\title{
Childhood malnutrition is associated with a reduction in the total melanin content of scalp hair
}

\author{
Colin A. McKenzie ${ }^{1 *}$, Kazumasa Wakamatsu ${ }^{2}$, Neil A. Hanchard ${ }^{1}$, Terrence Forrester ${ }^{1}$ and Shosuke Ito $^{2}$ \\ ${ }^{1}$ Tropical Metabolism Research Unit, Tropical Medicine Research Institute, University of the West Indies, Mona, \\ Kingston 7, Jamaica \\ ${ }^{2}$ Department of Chemistry, Fujita Health University School of Health Sciences, Toyoake, Aichi 470-1192, Japan
}

(Received 8 September 2006 - Revised 17 January 2007 - Accepted 18 January 2007)

\begin{abstract}
Childhood malnutrition is known to be associated with visible lightening of hair colour (hypochromotrichia). Nevertheless, no systematic investigations have been carried out to determine the biochemical basis of this change. We used an HPLC method to measure melanins in the scalp hair of thirteen Jamaican children, diagnosed as having primary malnutrition, during various stages of their treatment and after recovery. During treatment for malnutrition, a progressive decrease in total melanin content along the hair shaft from tip to root (root:tip ratio: 0.62 (SD 0.31)) was observed. This ratio was significantly different $(P=0.003)$ from the ratio observed among children sampled several months after discharge from hospital (0.93 (SD 0.23)) and among normal control children (0.97 (SD 0.12)). Thus, it appears that a decrease in melanin content is associated with periods of malnutrition. The low root:tip ratio during malnutrition presumably arises because the tips reflect prior hair growth during 'normal' nutrition and the roots reflect hair growth during malnutrition; a return of the root:tip ratio to that seen among controls reflects 'recovery' from malnutrition. It is possible that reduced intake or availability of tyrosine, a key substrate in melanin synthesis, may play a role in the reduction of hair melanin content during periods of malnutrition. The precise mechanisms by which melanin content is reduced, and the role of aromatic amino acid availability in hair colour change and other features of childhood malnutrition remain to be explored.
\end{abstract}

Eumelanin: Phaeomelanin: Hair: Childhood malnutrition

Severe childhood malnutrition is known to be associated with hypopigmentation of scalp hair (Bradfield \& Jelliffe, 1974; McLaren, 1987; Miller, 1989) and occasionally with the flag sign which consists of alternating areas of lighter and darker scalp hair so that there appear to be stripes of lightened hair running parallel to the scalp (Cline, 1988; Albers et al. 1993). The lighter-coloured bands of hair are thought to represent hair growth during periods of malnutrition (Golden, 1996) but the details of the cause of this sign and also of generalised hypopigmentation in malnutrition are unknown.

Two types of melanin pigment are produced in follicular melanocytes: eumelanin, a dark brown to black pigment, and phaeomelanin, a reddish-brown pigment (Ito, 2003). The production of melanin pigment proceeds from the conversion of L-tyrosine to dopaquinone, catalysed by tyrosinase, through a series of decarboxylation and oxidation steps which ultimately result in either eu- or phaeomelanin; melanin present in pigmented tissues consists of a mixture of these pigments (Hearing, 1998; Ito, 2003). The level of pigmentation in mammalian hair is under strong genetic control (Rees, 2003). In mice more than 130 genes are known to be involved in pigmentation (Bennett \& Lamoreux, 2003) and in man, there are many genetic disorders, including various types of oculocutaneous albinism, which cause hypopigmentation of scalp hair (Oetting et al. 2003). Hypopigmentation may also be a by-product of primary abnormalities in other metabolic pathways. For example, accumulation of alternative products of phenylalanine metabolism in phenylketonuria (Farishian \& Whittaker, 1980), and reduced availability of $\mathrm{Cu}$ in Menkes' syndrome (Tomita et al. 1992) may both result in hypopigmentation through secondary effects on melanin synthesis.

In the present paper we report on analyses of the total melanin content of scalp hair obtained from thirteen Jamaican children at different stages of their treatment for malnutrition. We examine the amount of melanin found at different points along the hair shaft, and compare the patterns among malnourished children with the patterns observed in samples from six normally nourished control children.

\section{Materials and methods \\ Participants \\ Cases. Cases were children who had been admitted to the metabolic research ward or had been referred to the Out- patients' Clinic of the Tropical Metabolism Research Unit (Jamaica), with a diagnosis of either marasmus $(<60 \%$ weight-for-age (W/A), no oedema), marasmic-kwashiorkor}


( $<60 \%$ W/A with oedema), underweight (60-80\% W/A, no oedema), or kwashiorkor (60-80\% W/A with oedema) according to the Wellcome Classification (Anonymous, 1970).

The research ward of the Tropical Metabolism Research Unit, Faculty of Medical Sciences, University of the West Indies is located at the University Hospital of the West Indies in the parish of St Andrew in the south-eastern end of Jamaica and serves as a tertiary referral centre for children with severe malnutrition from the entire island. Nutritional rehabilitation at the Tropical Metabolism Research Unit has been described in detail previously (Golden, 1993, 1996; McKenzie et al. 1998; Marshall et al. 2006). Briefly, on admission, acute problems are treated and children are started on a regimen which includes broad-spectrum antibiotics, multivitamin supplements, and milk-based 'maintenance' feeds $(418 \mathrm{~kJ}(100 \mathrm{kcal}) / \mathrm{kg}$ per d). Rapid catch-up growth follows and is achieved by offering high-energy feeds in an ad libitum fashion. The end of rapid catch-up growth is usually marked by a normal appetite and the attainment of the median weight-for-height. At this point, children are given a mixed diet appropriate for age and when established on the mixed diet, they are discharged from the ward and are usually seen as out-patients for 2 years post-discharge.

For this project, children with malnutrition were enrolled at different stages of their treatment. Patients recruited soon after admission to hospital or after referral to the out-patients' clinic contributed 'acute' samples. Patients recruited just before discharge from hospital contributed 'discharge' samples. Patients recruited several months after discharge from hospital contributed 'delayed' samples. At each stage that samples were obtained, W/A, weight-for-height and height-for-age were computed using the 1978 Centers for Disease Control and Prevention/WHO charts option in Epi Info ${ }^{\mathrm{TM}}$ (v. 3.3.2; Centers for Disease Control and Prevention, Atlanta, GA, USA) and are expressed as a percentage of the expected value.

Controls. We obtained hair samples from six healthy children (aged 14.0 (SD 5.0) months). These participants were recruited from among participants followed from birth in an ongoing longitudinal study that includes health surveillance at regular intervals (Thame et al. 2000).

The present study was approved by the University Hospital of the West Indies and University of the West Indies Faculty of Medical Sciences Ethics Committee, and written informed consent was given by a parent or adult guardian for each child before recruitment.

\section{Collection of hair samples}

First, a small tuft of hair (approximately $10 \mathrm{~mm}$ in diameter) was raised from the scalp of each participant (usually close to the occiput or bregma). This was removed by cutting the hair as close to the scalp as possible using a clean pair of scissors. The lower third of this sample was then taken as the 'root', the upper third taken as the 'tip', and the remainder was taken as the 'middle'. Where the length of hair allowed for it (children numbered 1, 2, 3 and 4), the hair sample was divided into equal $10 \mathrm{~mm}$ parts; the sample closest to the scalp was then taken as the root and the sample furthest from the scalp taken as the tip (the remainder was collectively deemed 'middle'). If the hair was very short, the hair was cut into two equal parts - root and tip.

\section{Determination of melanin content of hair samples}

Scalp hair samples from the participants were analysed for eumelanin and phaeomelanin content using methods which have previously been described in detail (Ito \& Fujita, 1985; Ito \& Wakamatsu, 1994; Ozeki et al. 1995, 1996; Wakamatsu \& Ito, 2002; Wakamatsu et al. 2002). Briefly, hair samples were homogenised in $0.5 \mathrm{ml}$ water with a Ten-Brocke glass homogeniser, and the resulting suspensions were then sonicated with an ultrasonic cell disrupter. Samples of $100 \mu \mathrm{l}$ were then treated with $\mathrm{KMnO}_{4}$ and $\mathrm{HI}$. These treatments result in the degradation of eumelanin and phaeomelanin to pyrrole-2,3,5-tricarboxylic acid and 4-amino-3-hydroxyphenylalanine respectively; these specific degradation products are analysed by HPLC. The assay for $\mathrm{KMnO}_{4}$ oxidation was performed in duplicate, and the mean of the duplicate readings is reported. The amount of eumelanin is obtained by multiplying the amount of pyrrole-2,3,5-tricarboxylic acid by 160 (Ozeki et al. 1996). The amount of phaeomelanin is obtained by multiplying the amount of 4-amino-3-hydroxyphenylalanine by 9 (Wakamatsu et al. 2002). All of the hair samples obtained in the present study were black to grey in appearance and contained predominantly $(>99 \%)$ eumelanin. Thus, in the present paper we report the combined amount of eumelanin and phaeomelanin as total melanin.

\section{Statistical methods}

Our principal objective was to determine whether there were significant between-group (acute, discharge, delayed, control) differences in total melanin content. Hair colour (melanin content), however, varies between individuals. In the present paper we use the root:tip ratio for between-group comparisons since this, effectively, controls for this variation. Unpaired $t$ tests were used in exploratory analyses to test for differences between acute and control samples and also to test for differences between delayed and control samples. An overall test of between-group differences was performed by fitting a robust linear regression model to the data. The robust model accounts for the fact that some participants contributed samples at two time points. All tests were carried out using Stata8 (StataCorp, College Station, TX, USA).

\section{Results}

\section{Participants}

Fourteen children with malnutrition were enrolled in the present study originally. A diagnosis of Bartter's syndrome was subsequently made in the case of one patient, however, and all the data from this patient were excluded from our analyses. Nine children (nos. 1, 2, 3, 4, 6, 7, 9, 11 and 13) contributed acute samples; six of these children had oedematous malnutrition whereas the others were underweight. The median duration of treatment at the time these samples were obtained was 3 (interquartile range 1-3) weeks. Two children (nos. 5 and 7) contributed discharge samples at 2.9 and 8 weeks post-admission respectively. Finally, seven children (nos. 1, 2, 3, 4, 12, 16 and 17) contributed delayed samples; the median elapsed time since either admission to hospital or first out-patient visit was $15 \cdot 2$ (interquartile range 9-17) months. Paired samples were thus available for five children (acute plus 
discharge samples for one child, and acute plus delayed samples for four children). Only unpaired samples were available for the remaining children.

Descriptive characteristics of participants at the times that samples were collected are given in Table 1. There were obvious differences in anthropometric values between the stages of treatment for malnutrition and the controls. For instance, as expected, children at the acute stage had the lowest W/A and weight-for-height values while the controls had the highest W/A and weight-for-height values.

\section{Total melanin content of scalp hair}

Hair samples were categorised as being from the 'tip', 'root' or the 'middle' of the hair shaft (see Materials and methods). The total melanin content of these hair samples is reported as the mean values and standard deviations. In hair samples from the six control children the total melanin content was 16.0 (SD 3.7) $\mu \mathrm{g} / \mathrm{mg}$ hair in the tips, 15.1 (SD 4.0) $\mu \mathrm{g} / \mathrm{mg}$ hair in the middle, and 15.3 (SD 3.0) $\mu \mathrm{g} / \mathrm{mg}$ hair in the roots. The root:tip ratio of melanin content was 0.97 (SD 0.12). Among the cases, in contrast, the total melanin content along the hair shaft varied with the stage of treatment. In the acute samples, obtained before children had undergone nutritional rehabilitation, nearly all patients showed a similar pattern of decreasing melanin content going from tip to middle to root (Fig. 1); there did not appear to be a marked difference between children (nos. 3, 4, 6 and 7) who had hair with dark tips and lightcoloured roots (i.e. a variant of the classical flag sign) and those children without any visible difference between tips and roots. The average melanin content in the tips was $30.1(\mathrm{SD} \mathrm{16} \cdot 4) \mu \mathrm{g} / \mathrm{mg}$ hair and in the roots was 15.6 (SD $6.7) \mu \mathrm{g} / \mathrm{mg}$ hair with a root:tip ratio of 0.62 (SD 0.31 ), which is significantly different $(t 2.37 ; P=0.021)$ from the root:tip ratio observed among samples from the controls.

Among patients who contributed either discharge or delayed samples, there was less uniformity of the melanin content along the hair shaft (Fig. 2). The majority of the children showed either an increase in total melanin going from tip to root (i.e. a reversal of the pattern seen in the acute samples) or showed a reduction in the tip to root gradient (i.e. root:tip ratios approaching 1) compared with the gradients seen among the acute samples. There were, however, several children (nos. 3, 4 and 7) who showed a marked decrease in melanin content going from tip to root which was similar to that seen among the acute samples. In the delayed samples the total melanin content in the tips was 19.2 (SD 5.1) $\mu \mathrm{g} / \mathrm{mg}$ hair and in the roots was 17.5 (SD 5.0) $\mu \mathrm{g} / \mathrm{mg}$ hair. The root: tip ratio was 0.93 (SD 0.23 ), which is not significantly different ( $t 0.38 ; P=0.709)$ from the root:tip ratio observed among the six control children.

Inspection of the root:tip ratios of samples collected at different stages (Fig. 3) suggests that in moving from 'acute' to discharge to 'delayed' time points, the root:tip ratios increase from relatively low values to values very similar to those of normal controls. A linear regression model, accounting for the fact that some of the cases contributed paired samples, was fitted to the data and confirmed that there was a significant difference $(P=0.013)$ between the root: tip ratio observed for the acute samples and the root:tip ratio observed among the delayed samples. There was also a significant difference $(P=0.008)$ between the root:tip ratio observed among the acute samples and the root:tip ratio observed among the controls. There was no significant difference in root:tip ratio between the acute and discharge samples $(P=0.46)$; this latter observation is unsurprising given that there were only two children sampled at discharge. Defining the point at which a child becomes undernourished is more difficult than for more severe forms of childhood undernutrition (where admission to hospital is a useful proxy for the onset of serious illness). We thus fitted the same linear model as above after excluding undernourished children. We found that the significance of the differences in root:tip ratios between acute and delayed samples $(P=0 \cdot 0001)$, between acute and control samples $(P=0 \cdot 002)$, between acute and discharge samples $(P=0 \cdot 13)$ and between control and delayed samples $(P=0 \cdot 23)$ were not materially different from analyses which included undernourished patients. It appears, therefore, that a decrease in melanin content in the roots compared with the tips (i.e. a low root:tip ratio) is associated with malnutrition whereas higher root:tip ratios are associated with the recovered state.

\section{Discussion}

It is well recognised that malnutrition is associated with both generalised hypopigmentation of scalp hair as well as the less common flag sign. Nevertheless, the biochemical basis of these changes in appearance has not been described previously. In the present study we have shown that there is a substantial negative gradient in total melanin content from the tips to the roots of hair shafts (root:tip ratio 0.62 ) at the

Table 1. Descriptive characteristics of participants by status at the time hair samples were obtained (Median values and interquartile ranges (IQR))

\begin{tabular}{|c|c|c|c|c|c|c|c|c|}
\hline & \multicolumn{2}{|c|}{ Acute } & \multicolumn{2}{|c|}{ Discharge } & \multicolumn{2}{|c|}{ Delayed } & \multicolumn{2}{|c|}{ Control } \\
\hline & Median & IQR & Median & IQR & Median & IQR & Median & IQR \\
\hline Subjects $(n)$ & \multicolumn{2}{|c|}{9} & \multicolumn{2}{|c|}{2} & \multicolumn{2}{|c|}{$r$} & \multicolumn{2}{|c|}{6} \\
\hline Male $(n)$ & \multicolumn{2}{|c|}{4} & \multicolumn{2}{|c|}{2} & \multicolumn{2}{|c|}{2} & \multicolumn{2}{|c|}{3} \\
\hline Age (months) & $11 \cdot 7$ & $8 \cdot 7,16 \cdot 5$ & $10 \cdot 7$ & $9.5,11.9$ & 24.4 & $17 \cdot 5,31 \cdot 6$ & $16 \cdot 0$ & $10 \cdot 9,19 \cdot 7$ \\
\hline $\mathrm{W} / \mathrm{A}^{*}$ & 64.4 & $45 \cdot 1,69 \cdot 4$ & $71 \cdot 2$ & $61.9,80.5$ & 70.5 & $63,95.4$ & $96 \cdot 6$ & $88 \cdot 1,102 \cdot 8$ \\
\hline $\mathrm{H} / \mathrm{A}^{*}$ & 89.4 & $77 \cdot 6,89 \cdot 8$ & 90.4 & $88.5,92 \cdot 2$ & 85.7 & $81 \cdot 1,100 \cdot 7$ & 99.5 & $96 \cdot 0,101 \cdot 9$ \\
\hline $\mathrm{W} / \mathrm{H}^{*}$ & 82.5 & $79 \cdot 0,89 \cdot 7$ & $89 \cdot 2$ & $82.9,95.4$ & 91.0 & $86 \cdot 2,100 \cdot 5$ & $95 \cdot 3$ & $89 \cdot 6,110 \cdot 4$ \\
\hline
\end{tabular}

W/A, weight-for-age; H/A, height-for-age; W/H, weight-for-height.

${ }^{*}$ Expressed as a percentage of the median value. 


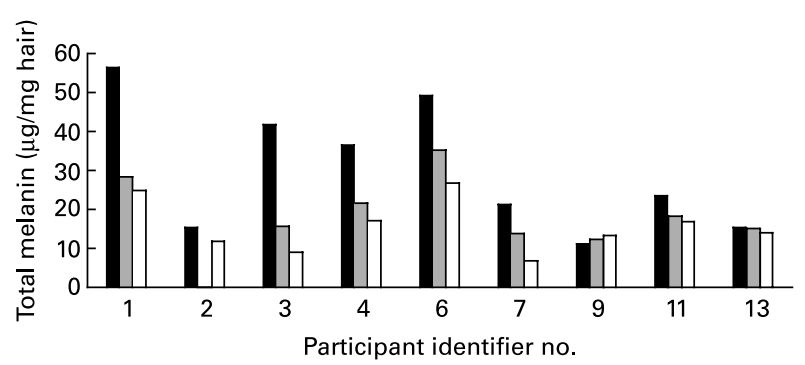

Fig. 1. Total melanin content of scalp hair obtained from malnourished chil-

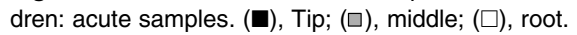

time of initiation of treatment for malnutrition. This ratio is significantly different from the root:tip ratio observed in children sampled several months after discharge from hospital and also from control children ( $P=0.013$ and 0.008 respectively). In the present study we have also shown that there is no significant difference between the root:tip ratio several months after discharge and the root:tip ratio among control children. For samples taken during the acute period, the melanin content of the tips probably reflects hair growth during the period before some critical change occurs in nutrient availability or enzyme activity, and the melanin content of the roots reflects hair growth after the change. Taken all together, the present data suggest that reduction in the total melanin content of scalp hair is a feature of malnutrition and is likely to account for the visual appearances of hair in malnourished children.

The precise sequence which leads to reduced melanin in the hair of malnourished children is, at present, unknown. Relatively poor availability of tyrosine may be a contributor. Tyrosine is essential for the production of melanin pigments, and levels of pigmentation in cultured melanocytes depend on tyrosine concentration (Hirobe et al. 2002, 2003). Tyrosine, a non-essential amino acid, can be derived from the essential amino acid phenylalanine, which is usually readily hydroxylated to form tyrosine by phenylalanine hydroxylase (Sandler \& Ruthven, 1969). In childhood malnutrition the combination of marginal or deficient intake and infection is common and might create an increased requirement for aromatic amino acids. The consequent increased rates of synthesis of positive acute-phase proteins, which contain relatively high proportions of aromatic amino acids, might explain why there is reduced or limiting availability of these amino acids (Reeds et al. 1994). It is possible that measurement of tyrosine

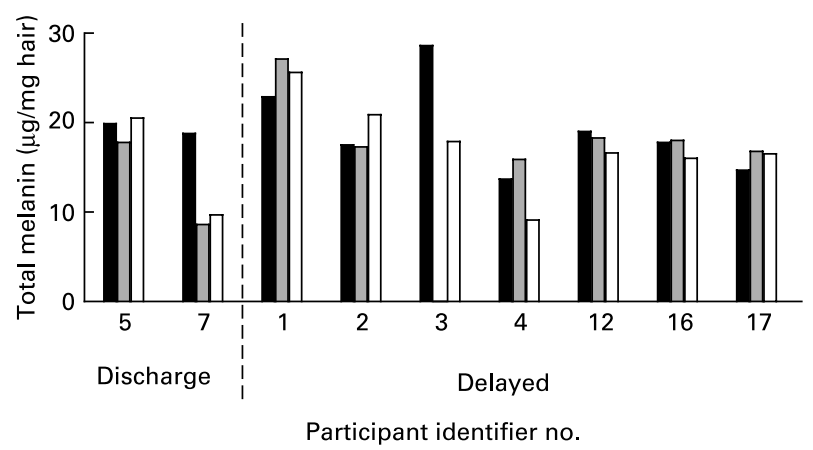

Fig. 2. Total melanin content of scalp hair obtained from children either at discharge from the ward or several months after discharge ('delayed' samples). ( $\square$ ), Tip; ( $\square$ ), middle; ( $\square$ ), root.

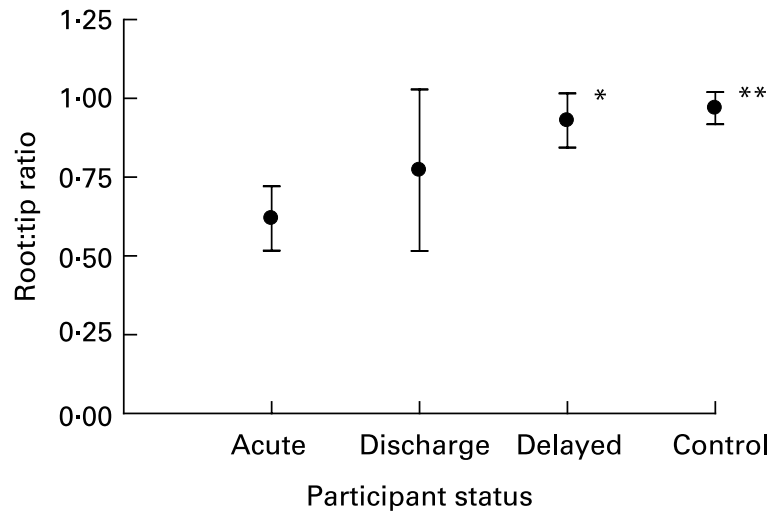

Fig. 3. Root:tip ratios of melanin among cases at different stages of treatment and among controls. Values are means, with $95 \% \mathrm{Cl}$ indicated by vertical bars. Mean value was significantly different from that of 'acute' samples: ${ }^{\star} P<0.05,{ }^{\star \star} P<0.01$ (two-sided).

concentrations (in serum, for example) at different times during the treatment of patients with malnutrition might be helpful in this regard; tyrosine concentrations were not measured in the present study.

Reduced tyrosinase activity might contribute to reduced melanin content of hair in malnutrition. When tyrosinase activity is low, cysteinyldopas are produced but pigment is not formed; at successively higher levels of tyrosinase activity, eumelanin production ultimately dominates (Ito, 2003). In Menkes' syndrome (Tomita et al. 1992) and in phenylketonuria (Farishian \& Whittaker, 1980), hypopigmentation is thought to be due to low or inhibited tyrosinase activity. Reduced availability of $\mathrm{Cu}$, although a possible cause of reduced tyrosinase activity (Petris et al. 2000), seems an unlikely contributor due to its estimated low prevalence among malnourished children in Jamaica (Golden et al. 1985). Reduced activity of phenylalanine hydroxylase is another possible contributor to reduced melanin content; reduced availability and/or utilisation of $\mathrm{Fe}$ in childhood malnutrition has been described (Golden et al. 1985; Waterlow, 1992) and it is known that Fe-deficiency anaemia is associated with impaired phenylalanine to tyrosine conversion (Lehmann \& Heinrich, 1986). It is thus possible that acquired phenylalanine hydroxylase deficiency may play a role in hair colour change in childhood malnutrition.

If a reduced root:tip ratio is due only to a change in the availability of nutrients during continuous hair growth, then hair samples obtained after recovery (either just before discharge or many months after discharge) would be expected to have root:tip ratios close to 1 since hair growth would have occurred under conditions of relatively constant nutrient availability. In this preliminary study, however, we found that there were several children who, many months after discharge from hospital, have tip to root gradients which resemble the acute pattern (albeit often of smaller magnitude). This finding might be explained by the persistence of the increased proportion of hairs in telogen which is known to occur in malnutrition (Bradfield, 1972; Bradfield \& Jelliffe, 1974), or by recurrent (unrecognised) bouts of malnutrition. Our clinical experience, however, is that after discharge from the ward the prevalence of serious nutritional problems is low (Barker et al. 2004) and that there is substantial hair growth indicating 
a return to normal anagen:telogen proportions. A more likely possibility is that the children in the present study returned to marginal rather than ideal nutritional intakes after discharge from hospital. Although there have been no reports on the effects of variation in the availability of tyrosine (and/or phenylalanine) on the colour of human hair, it has been demonstrated recently (Anderson et al. 2002) that in order for cats to maintain black fur colour they require dietary intakes of phenylalanine and tyrosine which are considerably in excess of the requirements for normal growth.

In a situation of stable but marginal intake there may be a gap between the rates of nutrient supply and those of utilisation for melanin deposition. Initiation of melanin deposition might be normal but subsequent rounds of deposition might exhaust the pool and a tip to root gradient might arise. In the present study, although we are unable to formally distinguish between the different possibilities, the fact that a substantial negative tip to root gradient persisted in one patient (no. 7) sampled just before discharge from the ward (i.e. after a period of closely supervised intake) provides some support for this notion. During treatment this child demonstrated a period of rapid catch-up growth which suggests that nutrient availability was sufficient to achieve weight gain but not sufficient for deposition of substantial amounts of hair melanin. Overall then, the present data are consistent with the idea that negative tip to root gradients might arise not only with changes (reductions) in intake but might also arise with stable, but marginal nutrient availabilities which are not low enough to be associated with severe weight loss.

It seems a reasonable proposition that reduced intake or availability of tyrosine and/or phenylalanine may play a role in the observed reduction of hair melanin content in malnutrition and we have proposed a model to explain the occurrence of tip to root gradients in situations of stable but marginal intakes. Despite the plausibility of the proposed model, the number of participants examined in the present study is small and the observed differences between acute and delayed samples may be due to random or systematic errors. For instance, no record was made of whether participants had hair cuts before sampling at the delayed stage; a hair cut just before sampling might be expected to result in a root:tip ratio of 1 . Only one child, however, had very short hair and this child still had a marked tip to root gradient; hair cuts would thus seem to be an unlikely explanation of the present findings. Nevertheless, the impact of sources of bias and the play of chance will need to be assessed by replicating our findings in larger datasets. If the hair shaft does capture a record of biochemical processes which have occurred before the onset of serious illness, our approach of making measurements at different points along the hair shaft might shed some light not only on hypopigmentation but might even give some insights into additional roles that determinants of melanin biosynthesis may have in childhood malnutrition.

\section{Acknowledgements}

Principally, we are grateful to the patients of the Tropical Metabolism Research Unit who participated in the present study. We also thank Dr Susanna Bortolusso-Ali, Nurses Kenesha Pennicott-Brown and Renée Walters, Mrs Karen
Aldred, Ms Joan Patterson, and Mr Alvan Brown for assistance in recruitment of participants. These data were presented in part at the 19th International Pigment Cell Conference, Reston, VA, USA, September 2005 and at the 51st Annual Conference of the Caribbean Health Research Council, St Kitts \& Nevis, West Indies, April 2006. The present study was supported in part, by a Grant-in-Aid for Scientific Research (no. 16591122) from the Ministry of Education, Culture, Sports and Technology of Japan.

\section{References}

Albers SE, Brozena SJ \& Fenske NA (1993) A case of kwashiorkor. Cutis 51, 445-446.

Anderson PJ, Rogers QR \& Morris JG (2002) Cats require more dietary phenylalanine or tyrosine for melanin deposition in hair than for maximal growth. $J$ Nutr 132, 2037-2042.

Anonymous (1970) Classification of infantile malnutrition. Lancet ii, 302-303.

Barker D, Younger N, MooSang M \& McKenzie CA (2004) HIV serostatus and recovery from severe childhood malnutrition. A retrospective matched case-control study. West Indian Med $J \mathbf{5 3}$, $89-94$.

Bennett DC \& Lamoreux ML (2003) The color loci of mice - a genetic century. Pigment Cell Res 16, 333-344.

Bradfield RB (1972) A rapid tissue technique for the field assessment of protein-caloric malnutrition. Am J Clin Nutr 25, 720-729.

Bradfield RB \& Jelliffe DB (1974) Letter: Hair-colour changes in kwashiorkor. Lancet i, 461-462.

Cline DJ (1988) Changes in hair color. Dermatol Clin 6, 295-303.

Farishian RA \& Whittaker JR (1980) Phenylalanine lowers melanin synthesis in mammalian melanocytes by reducing tyrosine uptake: implications for pigment reduction in phenylketonuria. J Invest Dermatol 74, 85-89.

Golden BE (1993) Primary protein-energy malnutrition. In Human Nutrition and Dietetics, pp. 440-455 [JS Garrow and WPT James, editors]. Edinburgh: Churchill Livingstone.

Golden MHN (1996) Severe malnutrition. In Oxford Textbook of Medicine, pp. 1278-1296 [DJW Weatherall, JGG Ledingham and DA Warrell, editors]. Oxford: Oxford University Press.

Golden MHN, Golden BE \& Bennett FI (1985) Relationship of trace element deficiencies to malnutrition. In Trace Elements in Nutrition of Children, pp. 185-207 [RK Chandra, editor]. New York: Nestlé Nutrition, Vevey/Raven Press.

Hearing VJ (1998) The regulation of melanin production. In The Pigmentary System. Physiology and Pathophysiology, pp. 423-438 [JJ Nordlund, R Boissy, VJ Hearing, RA King and J-P Ortonne, editors]. New York: Oxford University Press.

Hirobe T, Wakamatsu K \& Ito S (2003) Changes in the proliferation and differentiation of neonatal mouse pink-eyed dilution melanocytes in the presence of excess tyrosine. Pigment Cell Res 16, $619-628$

Hirobe T, Wakamatsu K, Ito S, Abe H, Kawa Y \& Mizoguchi M (2002) Stimulation of the proliferation and differentiation of mouse pink-eyed dilution epidermal melanocytes by excess tyrosine in serum-free primary culture. J Cell Physiol 191, 162-172.

Ito S (2003) The IFPCS presidential lecture: a chemist's view of melanogenesis. Pigment Cell Res 16, 230-236.

Ito S \& Fujita K (1985) Microanalysis of eumelanin and pheomelanin in hair and melanomas by chemical degradation and liquid chromatography. Anal Biochem 144, 527-536.

Ito S \& Wakamatsu K (1994) An improved modification of permanganate oxidation that gives constant yield of pyrrole2,3,5-tricarboxylic acid. Pigment Cell Res 7, 141-144. 
Lehmann WD \& Heinrich HC (1986) Impaired phenylalanine-tyrosine conversion in patients with iron-deficiency anemia studied by a L- $\left({ }^{2} \mathrm{H}_{5}\right)$ phenylalanine-loading test. Am J Clin Nutr 44, $468-474$.

McKenzie C, Boyne M, Venugopal S \& Cooper E (1998) Dumping syndrome in a young Jamaican child. West Indian Med $J \mathbf{4 7}$, 169-171.

McLaren DS (1987) Skin in protein energy malnutrition. Arch Dermatol 123, 1674-1676a.

Marshall KG, Howell S, Reid M, Badaloo AV, Farrall M, Forrester T \& McKenzie CA (2006) Glutathione S-transferase polymorphisms may be associated with risk of oedematous severe childhood malnutrition. Br J Nutr 96, 243-248.

Miller SJ (1989) Nutritional deficiency and the skin. J Am Acad Dermatol 21, 1-30.

Oetting WS, Fryer JP, Shriram S \& King RA (2003) Oculocutaneous albinism type 1: the last 100 years. Pigment Cell Res 16, 307-311.

Ozeki H, Ito S, Wakamatsu K \& Hirobe T (1995) Chemical characterization of hair melanins in various coat-color mutants of mice. J Invest Dermatol 105, 361-366.

Ozeki H, Ito S, Wakamatsu K \& Thody AJ (1996) Spectrophotometric characterization of eumelanin and pheomelanin in hair. Pigment Cell Res 9, 265-270.
Petris MJ, Strausak D \& Mercer JF (2000) The Menkes copper transporter is required for the activation of tyrosinase. Hum Mol Genet 9, 2845-2851.

Reeds PJ, Fjeld CR \& Jahoor F (1994) Do the differences between the amino acid compositions of acute-phase and muscle proteins have a bearing on nitrogen loss in traumatic states? J Nutr 124, 906-910.

Rees JL (2003) Genetics of hair and skin color. Аnпи Rev Genet 37, 67-90.

Sandler M \& Ruthven CR (1969) The biosynthesis and metabolism of the catecholamines. Prog Med Chem 6, 200-265.

Thame M, Osmond C, Wilks RJ, Bennett FI, McFarlane-Anderson N \& Forrester TE (2000) Blood pressure is related to placental volume and birth weight. Hypertension 35, 662-667.

Tomita Y, Kondo Y, Ito S, Hara M, Yoshimura T, Igarashi H \& Tagami H (1992) Menkes' disease: report of a case and determination of eumelanin and pheomelanin in hypopigmented hair. Dermatology 185, 66-68.

Wakamatsu K \& Ito S (2002) Advanced chemical methods in melanin determination. Pigment Cell Res 15, 174-183.

Wakamatsu K, Ito S \& Rees JL (2002) The usefulness of 4-amino-3hydroxyphenylalanine as a specific marker of pheomelanin. Pigment Cell Res 15, 225-232.

Waterlow JC (1992) Protein-Energy Malnutrition, pp. 58-60. London: Edward Arnold. 